\title{
Synaptic Reorganization in Scaled Networks of Controlled Size
}

\author{
Nathan R. Wilson, ${ }^{1 \star}$ Michael T. Ty, ${ }^{2 \star}$ Donald E. Ingber, ${ }^{2}$ Mriganka Sur, ${ }^{1}$ and Guosong Liu ${ }^{1,3}$ \\ ${ }^{1}$ Picower Institute for Learning and Memory and Department of Brain and Cognitive Sciences, Massachusetts Institute of Technology, Cambridge, \\ Massachusetts 02139, 2Department of Pathology, Children's Hospital and Harvard Medical School, Boston, Massachusetts 02115, and ${ }^{3}$ Center for Learning \\ and Memory, School of Medicine, Tsinghua University, Beijing 100084, China
}

Neurons in plastic regions of the brain undergo fundamental changes in the number of cells connecting to them as a result of development, plasticity and disease. Across these same time periods, functional changes in cellular and synaptic physiology are known to occur and are often characterized as developmental features of these periods. However, it remains possible that many such changes are direct consequences of the modified degree of partnering, and that neurons intrinsically scale their physiological parameters with network size. To systematically vary a recurrent network's number of neurons while measuring its synaptic properties, we used microfabricated extracellular matrix adhesive islands created with soft lithography to culture neuronal clusters of precise sizes, and assessed their intrinsic connectivity using intracellular recordings and confocal microscopy. Both large and small clusters supported constant densities of excitatory and inhibitory neurons. However, neurons that were provided with more potential partners (larger clusters) formed more connections per cell via an expanded dendritic surface than cocultured smaller clusters. Electrophysiologically, firing rate was preserved across clusters even as size and synapse number increased, due in part to synapses in larger networks having reduced unitary strengths, and sparser paired connectivity. Larger networks also featured a particular increase in the number of excitatory connections onto inhibitory dendrites. We suggest that these specific homeostatic mechanisms, which match the number, strength, and architecture of connections to the number of total available cellular partners in the network, could account for several known phenomena implicated in the formation, organization and degeneration of neuronal circuits.

Key words: synapse; network; inverse; quantal; homeostasis; inhibition

\section{Introduction}

A remarkable property of a neuron is its ability to sustain meaningful dynamics while the number of its functional cellular partners is being revised by development, refinement, plasticity, migration and disease. As an example, neurons in the developing lateral geniculate nucleus (LGN) across development reduce the number of retinal axon inputs with which they partner from $>20$ down to only one to three (Sur et al., 1984; Chen and Regehr, 2000). Similarly, during aging and pathological states such as Alzheimer's Disease, it is believed that brain areas such as the hippocampus suffer losses in neuronal number (Coleman and Flood, 1987; Simic et al., 1997). Throughout these changes, re-

\footnotetext{
Received Aug. 24, 2007; revised 0ct. 18, 2007; accepted 0ct. 22, 2007.

This work was supported by National Institutes of Health Grants EY15068 (M.S.) and CA55833 (D.E.I.) and National Basic Research Program of China Grants 2006 CB3031 and NSFC 30630026 (G.L.). N.R.W. was supported by a predoctoral fellowship from the National Science Foundation, and M.T.T. was supported by the Harvard-Massachusetts Institute of Technology Health Sciences and Technology Program. We thank the staff members in the Ingber laboratory for their technical assistance; members of the Sur and Liu laboratories for critical discussions and readings of this manuscript; and Robin Ty for the artistic rendering of the first figure. We dedicate this paper to the memory of Michael T. Ty.

*N.R.W. and M.T.T. contributed equally to this work.

Correspondence should be addressed to either of the following: Dr. Nathan R. Wilson, Massachusetts Institute of Technology, 46-6227, 77 Massachusetts Avenue, Cambridge, MA 02139, E-mail: nathan1@mit.edu; or Dr. Guosong Liu, School of Medicine, Tsinghua University, Beijing 100084, China, E-mail: liu.guosong@gmail.com. DOl:10.1523/JNEUROSCI.3863-07.2007

Copyright $\odot 2007$ Society for Neuroscience ～0270-6474/07/2713581-09\$15.00/0
}

maining neurons are believed to sustain comparable activity levels (Rao et al., 1993; Tanila et al., 1997; Smith et al., 2000), raising the question of whether changes in neuron number may directly impact the biophysics of network connectivity to sustain functional spiking as the size of the network scales.

Changes in synaptic physiology, for example, appear to correlate with alterations in partner number: neurons in the LGN exhibit a 50-fold increase in synaptic strength across development (Chen and Regehr, 2000). During aging, a decline in hippocampal circuitry may paradoxically be accompanied by increases to synaptic strength in those regions (Barnes and McNaughton, 1980; Foster et al., 1991). Still, despite the intuitive explanation that a neuron's synaptic circuitry might be responsive to the number of neurons available for partnering, an assay to highlight this relationship and distill which physiological parameters are involved has yet to be produced.

Assays for manipulating the number of neuronal partners have been implemented elegantly in muscle (Colman et al., 1997; Davis and Goodman, 1998; Lichtman and Colman, 2000; Davis and Bezprozvanny, 2001), where the number of input partners was genetically perturbed and the physiological consequences measured. In contrast, it is difficult to imagine a CNS preparation for cleanly varying the number of a neuron's partners, and the architecture is further complicated by the addition of recurrent connections and the interplay of excitatory and inhibitory con- 
nections. As such, although a relationship between the number of available partners and consequent synaptic physiology has been suggested (Liu and Tsien, 1995a), it has yet to be systematically studied.

We therefore sought to develop a simplified assay to (1) systematically vary the number of CNS neurons available in a network with recurrent connectivity, (2) examine the consequences of such perturbations for cellular and synaptic physiology, and (3) visualize excitatory and inhibitory connectivity patterns that change with network size. We adapted neuronal cultures to a soft lithography-based microcontact printing system (Singhvi et al., 1994; Whitesides et al., 2001) to engineer discrete neuronal networks in "islands" of distinct sizes but constant densities, on the same coverslip, at the same developmental stage, and in the absence of exogenous input. Our observations indicate that neurons, when connected recurrently and in the absence of external cues, can sense the size of the distributed cellular collective in which they are situated, and they endogenously redistribute synaptic efficacy to reflect this size.

\section{Materials and Methods}

Micropattern fabrication. AutoCAD software (Autodesk, San Rafael, CA) was used to define a spatial pattern template of increasingly larger squares. This pattern was custom printed onto a transparency (Advance Reproductions Corporation, North Andover, MA) and used to selectively cover regions of a silicon wafer covered with a thin layer of photoresist during UV exposure (Silicon Sense, Nashua, NH). Regions exposed to the UV hardened, whereas the regions covered by the pattern remained soft and eventually were washed away. The result was a threedimensional imprint of the original pattern designed in the software. This surface then served as a mold on which a polymer was poured, to create a "stamp" with microfeatures. Polydimethylsiloxane (PDMS; Dow Chemical, Midland, MI) was used as a polymer to coat the silicon surface, after which it was cured with heat. When the PDMS had solidified, it was gently peeled from the wafer mold and was used to bring the chemicals described below into contact with the surface of culturing interest.

To prepare the culture surface, an electron-beam evaporator (Massachusetts Institute of Technology Whitehead Institute) was first used to deposit a thin layer of gold (Sigma, St. Louis, MO; catalog \#373168) and titanium (Sigma, catalog \#433675) onto the surface of a glass coverslip. The PDMS stamp described above was then "inked" with hydrophobic alkanethiols dried with nitrogen gas, and brought into tight contact with the surface of the coverslip. Thus, alkanethiol molecules were transferred onto the gold and titanium surface in the predefined pattern of squares illustrated previously. Once the alkanethiols were deposited, they immediately formed self-assembled monolayers (SAMs) limited to the square islands.

Up to $12 \mathrm{~h}$ after alkanethiol deposition, patterned coverslips were sterilized in ethanol, rinsed several times in sterile water, and coated with laminin (50 $\mu \mathrm{g} / \mathrm{ml}, \mathrm{L} 2020$; Sigma). Laminin only adhered to the islands coated with the hydrophobic alkanethiol, and cell adhesion was therefore restricted only to these regions (Singhvi et al., 1994). When hippocampal neurons were plated onto the laminin-coated micropatterned islands, they preferentially attached to these islands as well as avoided the gold surface. Over the next $4 \mathrm{~d}$, approximately, the adherence between neuron and original island shape tightened as neurons settled and dispersed their axons and dendritic fields to assume each island's size and shape.

Cell culture. Hippocampi were dissected from postnatal day 1 (P1) Sprague Dawley rat pups and cultured as described previously (Liu and Tsien, 1995b). Measurements of pattern structure were performed at $14 \mathrm{~d}$ in vitro (DIV), miniature EPSC (mEPSC) recordings at DIV 15 (replicated as late as DIV 19), evoked transmission at DIV 18, spontaneous transmission and spiking at DIV 14-18, and all quantitative immunohistochemistry experiments at DIV 14-15. All experiments involving animals were approved by the Massachusetts Institute of Technology's Committee on Animal Care.

Imaging. Fluorescent imaging of synaptic puncta and dendritic branches was performed using an Olympus (Melville, NY) Fluoview confocal microscope with a $10 \times, 20 \times$, or $40 \times$ plan-apochromatic waterimmersion lens (1.15 numerical aperture). Cell cultures were fixed in $4 \%$ paraformaldehyde and $4 \%$ sucrose in PBS and permeabilized with $0.5 \%$ Triton X-100 for $30 \mathrm{~min}$ at $22-24^{\circ} \mathrm{C}$. Primary antibodies against microtubule-associated protein 2 (MAP2; Millipore, Temecula, CA), synapsin I (American Qualex, San Clemente, CA), $\alpha$-calciumcalmodulin-dependent protein kinase ( $\alpha$-CaMKII; Zymed, San Francisco, CA; Invitrogen, Carlsbad, CA), vesicular glutamate transporter 1 (VGLUT1; Millipore), vesicular GABA transporter (VGAT; Alpha Diagnostics, San Antonio, TX), or GABA (Sigma) were applied, followed by rinses in PBS and visualization with Alexa 488-, 546-, or 633-conjugated secondary antibodies (1:400; Invitrogen). All images were collected at $1280 \times 1024$ pixel resolution and $1-4 \times$ software zoom, using a $z$ series projection of $8-11$ images taken at $0.8 \mu \mathrm{m}$ depth intervals. Single-cell labeling (see Fig. $3 E$ ) was achieved by backfilling a pipette with the intracellular solution described below plus Alexa 488 dye, and then waiting for $20 \mathrm{~min}$ after rupture to image the filled cell.

Electrophysiology. Patch pipettes (2-6 $\mathrm{M} \Omega$ ) contained (in $\mathrm{mm}$ ) potassium 120 gluconate, $3 \mathrm{KCl}$, 10 HEPES, $8 \mathrm{NaCl}, 0.5 \mathrm{CaCl}_{2}$, 5 EGTA, 2 $\mathrm{Mg}^{2+}$-ATP, and $0.3 \mathrm{GTP}, \mathrm{pH}$ adjusted to 7.3 with $\mathrm{NaOH}$. Extracellular solution was based on Tyrode's solution containing (in mM) $145 \mathrm{NaCl}, 3$ $\mathrm{KCl}, 15$ glucose, 10 HEPES, 1.3-2.6 $\mathrm{MgCl}_{2}, 1.3-2.6 \mathrm{CaCl}_{2}$, $\mathrm{pH}$ adjusted to 7.4 with $\mathrm{NaOH}$. EPSCs and mEPSCs were recorded under voltage clamp $(-60 \mathrm{mV})$ in the presence of $50 \mu \mathrm{M}$ picrotoxin (Sigma) using a MultiClamp 700A amplifier (Molecular Devices, Foster City, CA), sampled at $10 \mathrm{kHz}$, filtered at $1 \mathrm{kHz}$, and recorded using Axon AxoScope or in-house software written in $\mathrm{C}++$ (G. Liu laboratory). A $5 \mu \mathrm{M}$ concentration of 1,2,3,4-tetrahydro-6-nitro-2,3-dioxo-benzo[f] quinoxaline-7-sulfonamide (NBQX; Sigma) was used to verify AMPA currents. One micromolar tetrodotoxin (TTX; Biotium, Hayward, CA) was added for mESPC recordings. For dual-cell evoked EPSCs, a second whole cell patch was achieved and action potentials were stimulated by injecting 100-300 pA of current over $<10 \mathrm{~ms}$ (current clamp), or $30-80 \mathrm{mV}$ over $0.5 \mathrm{~ms}$ (voltage clamp).

Analysis. Quantification of electrophysiological data were performed in MiniAnalysis (Synaptosoft, Leonia, NJ) for mEPSCs, and custom scripts written in $\mathrm{C}++$ and Matlab (MathWorks, Natick, MA) for EPSCs evoked presynaptically. Traces for which access resistance varied substantially or were $>25 \mathrm{M} \Omega$ were rejected from analysis. mEPSCs were measured using a $4 \mathrm{pA}$ threshold in MiniAnalysis and then verified manually. Action potentials and EPSCs were located and quantified using custom MATLAB scripts and then verified manually. Image analysis was performed via custom scripts written in ImagePro Plus (Media Cybernetics, Carlsbad, CA) for puncta localization and quantification. Pixels within each punctum were assigned intensity values, and the combined sum of a punctum's intensity values, or integrated optical density, was taken as the punctum's total intensity. All error bars report standard mean error unless otherwise noted. All $p$ values refer to unpaired $t$ tests unless otherwise noted $\left({ }^{\star} p<0.05 ;{ }^{* *} p<0.01 ;{ }^{* *} p<0.001\right)$.

\section{Results}

\section{A system for designing the geometry of cultured neuronal networks to grow identical networks of varying size}

To explore specific changes in synaptic function that accompany changes in network architecture, we sought a system that would let us spatially restrict the size and shape of neuronal networks in an open-faced culture preparation amenable to direct optical and electrical measurements of function. We made use of cellular patterning methods afforded by microcontact printing (Chen et al., 2000; Whitesides et al., 2001), integrating fine-scale techniques developed by others (Scholl et al., 2000; Dertinger et al., 2002; Sanjana and Fuller, 2004) to our established culture protocols for quantifying synaptic physiology in neuronal cultures.

A micropattern was designed in AutoCAD and manifested as a three-dimensional PDMS stamp (see Materials and Methods) that matched the size and shape of our desired neuronal islands, 


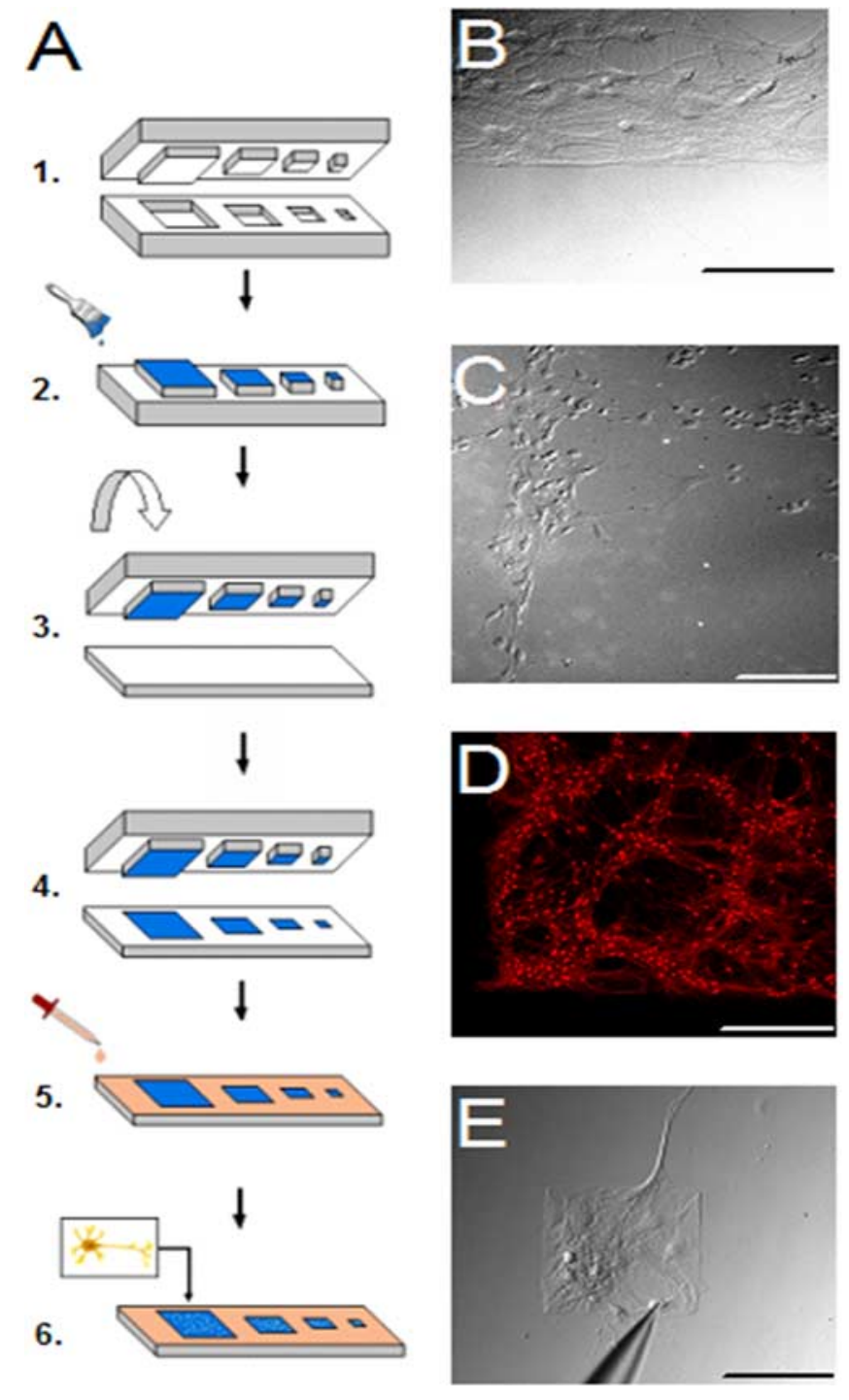

Figure 1. A system for specifying the geometry of cultured neuronal networks. $\boldsymbol{A}$, Schematic of method for generation of micropatterned cultures. 1, Features of photoresist are carved by ultraviolet light to create a mold, onto which PDMS is poured, cured by heat, cooled, and peeled from the wafer, to form a microstamp. 2, The microstamp is inked with purified hydrophobic alkanethiols and dried with nitrogen gas. 3, The stamp is brought down tightly on a coverslip, which has previously been covered with a thin layer of gold and titanium using an electronbeam evaporator. 4, Consequently, the hydrophobic alkanethiol molecules are transferred onto the regions of the glass surface that contacted the raised regions of the stamp, where they immediately formed self assembled monolayers. A solution of laminin is then applied, the physiosorption of which is specific to the patterned hydrophobic regions. 5, PEG is applied and binds preferentially to the nonpatterned regions, to further repel neurons. 6, Finally, hippocampal neurons are dissected, dissociated and plated onto the laminin coated micropatterned substrates, preferentially attaching to the islands and spreading to assume the island's size and shape. $\boldsymbol{B}$, Neurons took root and eventually exhibited morphology similar to that observed in normal dissociated cultures. $\boldsymbol{C}$, Result of micropatterning neurons in a series of crossing lines. $\boldsymbol{D}$, Corner of a square microisland of neurons labeled with the excitatory neuron marker $\alpha$-CaMKII. $\boldsymbol{E}$, Example of how small the islands can be crafted to support neurons. Islands this small were not used in the remaining experiments. Scale bars: $\boldsymbol{B}, \boldsymbol{C}, \boldsymbol{E}, 200 \mu \mathrm{m} ; \boldsymbol{D}, 500 \mu \mathrm{m}$.

which we engineered to be small squares of geometrically increasing size. The remainder of the procedure is detailed graphically in Figure $1 A$. The stamp was "inked" with purified hydrophobic alkanethiols, and brought down tightly on a "coverslip," which had previously been covered with a thin layer of gold and titanium. A solution of laminin was then applied, the physiosorption of which was specific to the patterned hydrophobic regions. Poly- ethylene glycol (PEG) was next applied to bind preferentially to the nonpatterned regions, to further repel neurons. Finally, hippocampal neurons were dissected, dissociated and plated onto the laminin-coated micropatterned substrates, preferentially attaching to the islands and spreading to assume the island's size and shape.

After $14 \mathrm{~d}$ of incubation to allow circuits to mature within each network size, the stability of neurons on the substrates was assessed. Neurons took root and eventually exhibited morphology similar to that observed in normal dissociated cultures (Fig. $1 B$ ), and adhered closely to the template pattern, whether the design was for thin edges (Fig. 1C) or square islands (Fig. 1D,E). Several days were required for crisp pattern adherence: high fidelity was not observed until $\sim 4 \mathrm{~d}$ in culture (data not shown).

\section{Patterned networks of controlled size can be cocultured and} exhibit robust structural and functional properties

To explore how functional properties of cultured neurons changed based on the number of cellular partners available in their local network, we wished to create identical culture islands of varying size on the same coverslip. A template was thus created that featured increasingly larger squares, scaling in diameter from $200,600,1200$, to $2000 \mu \mathrm{m}$. Neurons plated on the chips manufactured from this template adhered with high fidelity, as captured by confocal imaging of P14 neurons fixed and stained with the excitatory neuronal marker $\alpha$-CaMKII (Fig. $2 A$ ).

Neurons isolated to squares were first identified as $4^{\prime}, 6$ diamidino-2-phenylindole (DAPI)-labeled cell bodies stained for the somatodendritic marker MAP2 (Fig. 2 B). Additional staining revealed a heterogeneous mix of DAPI-labeled cells with and without coexpression of MAP2 and the synaptic marker VGLUT1 (Fig. 2C), indicating that both neurons and nonneuronal glial support cells were present and that excitatory connections had formed between the neurons. The presence of synaptic connections was further elucidated with the presynaptic marker synapsin I (Fig. 2 D), which appeared as localized puncta. Synapses were verified as functional using the optical marker FM1-43 (Betz and Bewick, 1992) (Fig. 2E), although vesicle turnover in our inverted microscope system could only faintly be visualized through the opaque gold coverslips.

Island size, measured using $\alpha$-CaMKII staining and image reconstruction, was controlled quite rigorously with only small deviations in size across cultures (Fig. $2 F$ ). Cell density also tended to be quite stable regardless of the amount of island space available to the neurons for growth (Fig. $2 G$ ), perhaps because cells were deposited simultaneously and evenly across all islands followed by preferential cell loss in the nonadhesive regions.

Neurons were also immunolabeled with $\alpha$-CaMKII and GABA to verify the presence of excitatory and inhibitory cell bodies and dendrites (Fig. $2 \mathrm{H}$ ). Whereas hippocampal excitatory neurons are believed to prominently display $\alpha$-CaMKII and sometimes express GABA as well (Sloviter et al., 1996), inhibitory interneurons express GABA only and lack $\alpha$-CaMKII (Jones et al., 1994; Liu and Jones, 1996; Sik et al., 1998). We thus used an $\alpha$-CaMKII expression signature to differentiate excitatory neurons from GABA-positive inhibitory ones. Cell bodies could be visualized as bright puncta relative to diffuse dendritic staining, and the occurrences of these somata were counted manually. As shown in Figure 2I, a similar ratio of excitatory and inhibitory neurons tended to be present across island sizes (for assessments of neuron morphology and connectivity between different cell types, see below). 


\section{Changing network size changes structural synaptic capacity}

To distinguish whether cultured networks of different sizes result in a different or conserved number of synaptic connections per neuron, we performed immunostaining for synapsin I (Fig. 3A). For this and the remaining quantifications, we focused our measurements on the largest island (Fig. $2 \mathrm{~A}$, island 4 with $2 \mathrm{~mm}$ side length, "large") versus a smaller island (Fig. 2A, island 2 with 0.6 mm side length, "small"). Here, although neuron density was similar between large and small islands (Fig. 2G), synaptic density was dramatically increased on the larger island (1072 \pm 73 synapses/image, large vs $506 \pm 34$ synapses/image, small; $p<0.001$ unpaired $t$ test; $n=7$ images, large; 6 images, small) (Fig. 3C), indicating that neurons in the larger networks form more synapses with one another. This capacity for greater connectivity may be partially provided by somewhat larger dendritic trees per neuron, as the overall density of dendrites in the images (Fig. 3B) was also increased for large networks ( $42 \pm 3 \%$ pixels/image, large vs $24 \pm$ $4 \%$ pixels/image, small; $p=0.003$ unpaired $t$ test; $n=15$ images, large; 13 images, small) (Fig. 3D). Measured more directly, the extent of dendritic arborization measured per neuron by filling individual cells with Alexa 488 (Fig. 3E) was significantly longer in larger clusters for the primary "basal" dendrite (1821 \pm 167 pixels long, large vs $1274 \pm 117$ pixels long, small; $p=0.02$ unpaired $t$ test; $n=6$ cells, large; 8 cells, small) (Fig. $3 F$ ) and to some (nonsignificant) degree for branching "distal" dendrites $(2558 \pm 505$ pixels in length, large vs $1625 \pm$ 539 pixels in length, small; $p=0.22$ unpaired $t$ test; $n=6$ cells, large; 8 cells, small) (Fig. $3 F)$.

\section{Implications of increased connectivity for functional activity}

Measuring electrophysiological properties across micropatterned islands of different sizes, our first observation was that neurons on different islands exhibited comparable functional synaptic and spiking activity (Fig. $4 A, B$ ). First, synaptic activity was measured in voltage clamp (Fig. $4 A$ ), to establish the amount and distribution of excitatory input received by representative neurons. Second, spiking activity was measured in current clamp (Fig. $4 B$ ) to examine the actual integration of input and summed output by the neurons. The frequency of excitatory bursting was slightly (although not significantly) reduced in the smaller networks compared with the larger ones $(2.89 \pm 1.46$ $\mathrm{Hz}$, large vs $1.68 \pm 0.55 \mathrm{~Hz}$, small; $p=0.49$ unpaired $t$ test; $n=4$ cells, large; 4 cells, small) (Fig. $4 C$ ), an observation that might be anticipated because there were fewer synaptic partners to initiate and propagate bursting activity. However, the size of these bursts when they did occur was slightly (although not significantly) enhanced $(40.2 \pm 4.4 \mathrm{pA}$, large vs $56.1 \pm 10.5 \mathrm{pA}$, small; $p=0.24$ unpaired $t$
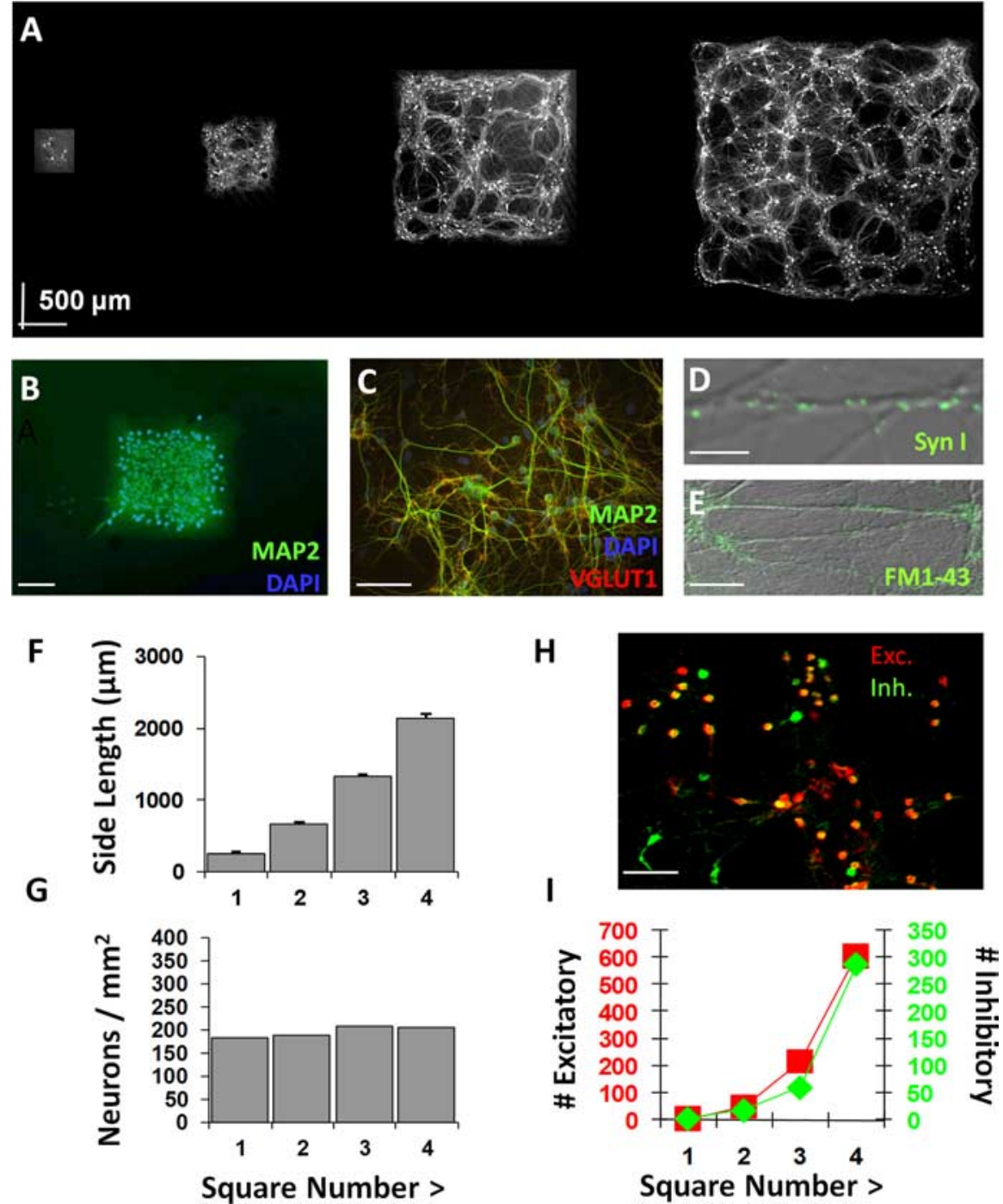

Figure 2. Neuronal islands of systematically varied size exhibit robust functional properties. $A$, Micropatterned template used in experiments. Neurons labeled with somatic and dendritic marker $\alpha$-CaMKII and imaged via confocal microscopy at $10 \times$. Manually montaged images made it possible to clearly delineate borders where cells and dendritic networks cannot grow. $\boldsymbol{B}$, solated squares contain neurons identified by DAPI-labeled cell bodies stained for the neuronal marker MAP2. C, Highermarker VGLUT1, indicating that both neurons and non-neuronal cells are present and synaptic connections have formed between neurons. $\boldsymbol{D}$, The presence of synaptic connections elucidated with the presynaptic marker synapsin I, which appears as localized side length showed little deviation across cultures. $\boldsymbol{G}$, Neuron cell density remained stable across squares of the same chip. $\boldsymbol{H}$, mmunolabeling with $\alpha$-CaMKII and GABA to label the full extent of excitatory and inhibitory cell bodies and dendrites. Cell bodies are visualized as bright puncta. $I$, A similar ratio of excitatory and inhibitory neurons tended to be present across island sizes. Scale bars: $\boldsymbol{B}, 500 \mu \mathrm{m} ; \boldsymbol{C}, \boldsymbol{H}, 100 \mu \mathrm{m} ; \boldsymbol{D}, 10 \mu \mathrm{m} ; \boldsymbol{E}, 20 \mu \mathrm{m}$. Error bars indicate SEM.

test) (Fig. 4D), such that the average integrated area of depolarization received by neurons in the different islands across time was nearly identical $(3.10 \pm 1.77 \mathrm{pA} / \mathrm{ms}$, large vs $3.90 \pm 1.05 \mathrm{pA} / \mathrm{ms}$, small; $p=0.71$ unpaired $t$ test) (Fig. $4 E$ ). Similarly, the median interval between action potentials was nearly identical across neighboring clusters of disparate size $(3.45 \pm 0.79 \mathrm{~s}$, large vs $3.66 \pm 0.98 \mathrm{~s}$, small; $n=12$ cells, large; 8 cells, small; $p=0.88$ unpaired $t$ test) (Fig. $4 F)$. Thus, neurons in islands of different sizes exhibited stable degrees of excitatory input and integrated output.

\section{Scaling network size changes unitary strength per synapse}

The fact that a small number of neurons with a reduced number of synapses consistently fires at the same rate as a large number of 
A

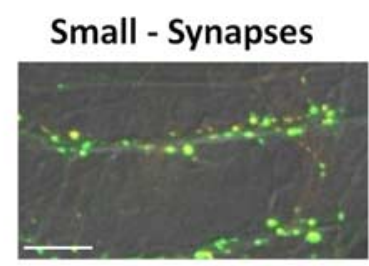

B

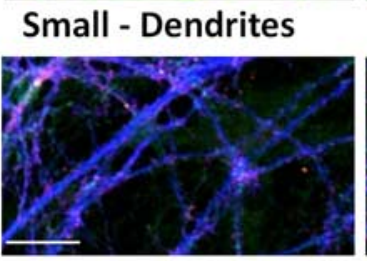

C
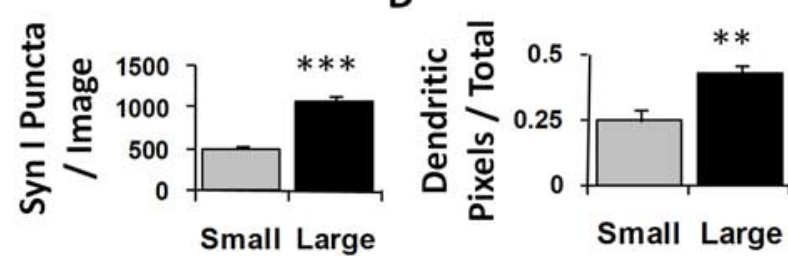

E

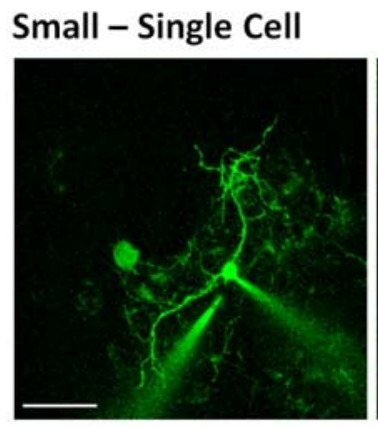

$\mathbf{F}$

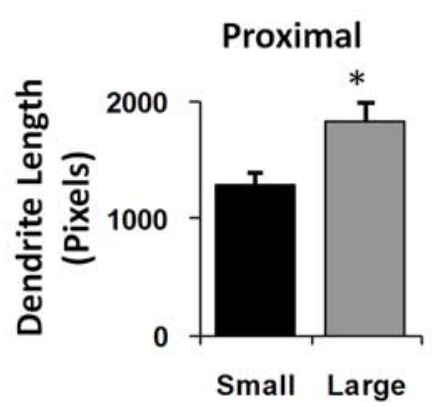

Large - Synapses

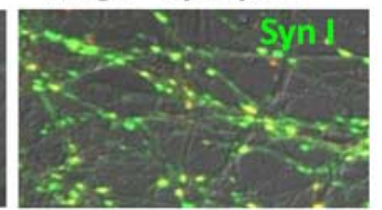

Large - Dendrites

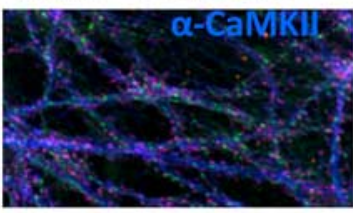

A

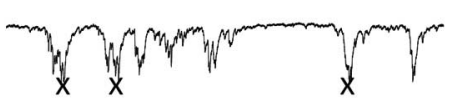

Large

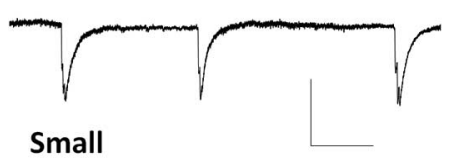

B
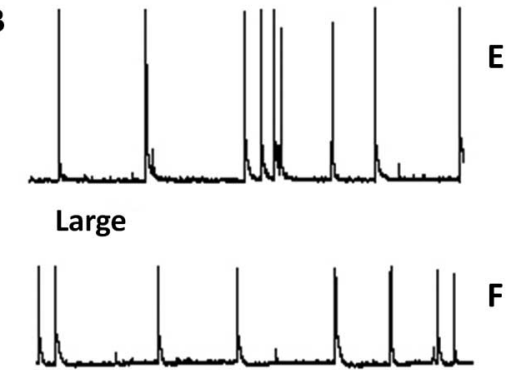

Small

$\mathbf{E}$
C

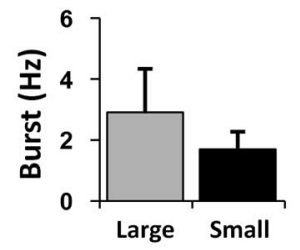

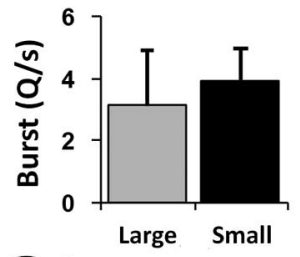

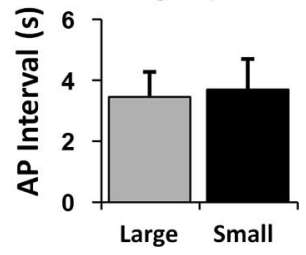

Figure 4. Implications of increased connectivity for functional activity. $A$, Excitatory synaptic bursting activity recorded intracellularly in voltage clamp for neurons in large islands (top traces) and small islands (bottom traces). Xs indicate the locations of transient spiking events that are omitted from traces to conserve space. $\boldsymbol{B}$, Action potentials recorded intracellularly in current clamp for neurons in large islands (top traces) and small islands (bottom traces). $\boldsymbol{C}$, Measuring the frequency of excitatory synaptic bursts revealed a slight but nonsignificant decrease in burst frequency in clusters comprising fewer neurons. $\boldsymbol{D}$, Measuring the amplitude of excitatory synaptic bursts revealed a slight but nonsignificant increase in peak amplitude for clusters comprising fewer neurons. $E$, Measuring the summed excitatory depolarization per unit time reveals no change in the total excitatory input to neurons situated in clusters of varying size. $\boldsymbol{F}$, Neurons across a variety of micropatterned islands also exhibit comparable functional activity in terms of action potential frequency. Calibration: $\boldsymbol{A}, 80 \mathrm{pA}, 400 \mathrm{~ms} ; \boldsymbol{B}, 100 \mathrm{mV}$, $4 \mathrm{~s}$. Error bars indicate SEM.

from the transmission of single vesicles. Plotting the distribution of quantal responses observed in large vs. small networks revealed that mEPSC responses recorded in small networks had significantly higher amplitudes than those recorded in large networks $(~ p<0.001$ Kolmogorov-Smirnov test; large, $n=1985$ mEPSCs analyzed from 14 cells; small, $n=2942$ events analyzed from 11 cells) (Fig. 5B). Overall, when averaged cell by cell, excitatory quantal size in small networks was markedly increased compared with mEPSC amplitude in large networks $(15.8 \pm 1.69 \mathrm{pA}$, large vs $24.51 \pm 1.96 \mathrm{pA}$, small; $p=0.003$ unpaired $t$ test; large, $n=14$ cells; small, $n=11$ cells) (Fig. $5 C$ ). However, mEPSC frequency was not observed to be significantly different in the two types of network $(4.01 \pm 1.14 \mathrm{~Hz}$, large vs $4.52 \pm 0.78$, small; $p=0.73$ unpaired $t$ test) (Fig. 5D), despite a reduced number of synapses in the small network (Fig. $3 A$ ).

Scaling network size changes the relative coupling between pairs of neurons

We next measured the extent of functional connectivity between pairs of synaptic partners, via evoked synaptic transmission using dual intracellular patch clamp (Fig. 5E). Action potentials were elicited in one "presynaptic" neuron while the synaptic response 
A
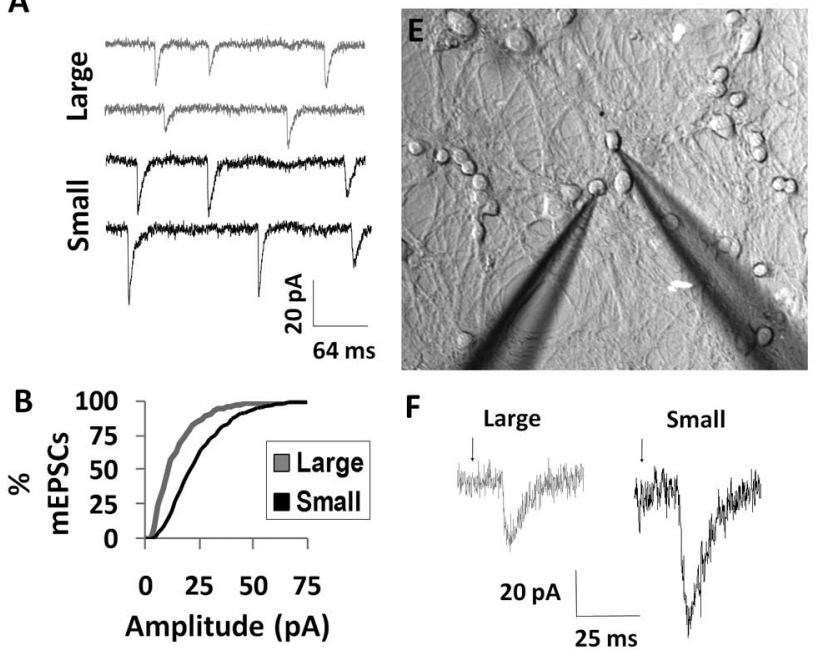

$F$
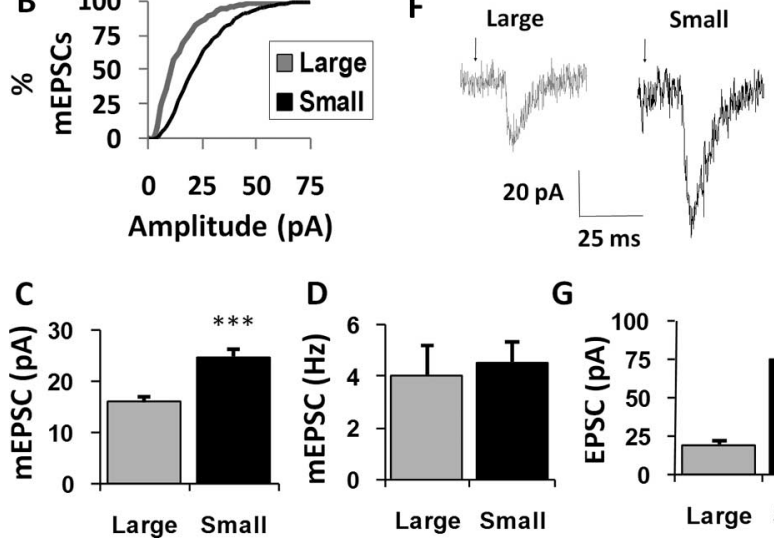

$\mathbf{G}$

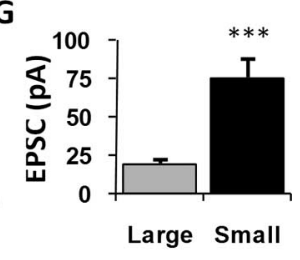

Figure 5. Scaling network size changes unitary strength per synapse and the functional coupling between pairs of neurons. $\boldsymbol{A}, \mathrm{mEPSC}$ were recorded postsynaptically via whole-cell patch clamp in the presence of TTX in either a large or small network. Calibration: 20 pA, 64 ms. $\boldsymbol{B}$, Cumulative density functions depicting distributions of $\mathrm{mEPSC}$ amplitudes recorded from large and small networks. A rightward shift is observed in mEPSC amplitudes recorded from small networks. C, Average mEPSC amplitudes for neurons recorded in each size network show an increase in mean event amplitude in small networks relative to larger ones. $\boldsymbol{D}$, Average $m E P S C$ frequency for neurons recorded in each size network. mEPSC frequency was not significantly different in the two types of networks, despite a reduced number of synapses in the small network (Fig. 3C). $\boldsymbol{E}$, The extent to which pairs of neurons were functionally coupled was assessed via evoked synaptic transmission using dual intracellular patch clamp. Action potentials were elicited in one "presynaptic" neuron whereas the synaptic response was measured in voltage clamp in the second "postsynaptic" neuron. $\boldsymbol{F}$, Typical evoked transmission (stimulus artifacts replaced with arrows) in small networks was significantly increased in amplitude compared with evoked transmission in large networks. G, Quantification for all evoked transmission in large and small networks. Evoked responses in small networks were significantly larger than evoked responses in large networks. Note that pairs where evoked transmission in one neuron triggered an action potential in the postsynaptic neuron were omitted, because such depolarizations were too large to quantify in voltage clamp with our patch circuits. Although presynaptically induced spiking was rare in large networks, it was relatively common in small networks. ${ }^{* *} p<0.001$. Error bars indicate SEM.

was measured in voltage clamp in the second "postsynaptic" neuron. Typical evoked transmission in large networks (Fig. 5F, left trace) was significantly reduced compared with similar transmission in small networks (Fig. $5 F$, right trace), a trend that was maintained across multiple paired connections $(18.9 \pm 2.92 \mathrm{pA}$, large vs $74.53 \pm 12.86 \mathrm{pA}$, small; $p=0.02$ unpaired $t$ test; large, $n=11$ pairs; small, $n=5$ pairs) (Fig. $5 G$ ). Evoked synaptic activity in some pairs overcame the voltage clamp in the postsynaptic neuron because of the large amplitude of the synaptic drive, and these pairs were not quantified. This was disproportionately common in the small networks (data not shown).

\section{Excitatory neurons amplify inhibition via innervation of inhibitory dendrites}

To test for changes in the identity and targeting of excitatory and inhibitory connections that occurred in networks of different sizes, we first used $\alpha$-CaMKII staining to restrict our analysis to excitatory dendrites. We then used the markers VGLUT1 (excitatory) and VGAT (inhibitory) to label the synapse type onto these excitatory dendrites in large vs. small networks (Fig. 6A). We observed that the ratio of excitatory and inhibitory synapses along a given length of excitatory dendrite was maintained at a constant level from one size network to another $(5.34 \pm 0.68$ large vs $4.44 \pm 0.84$ small; $p=0.43$ unpaired $t$ test; large, $n=5$ images, 4914 VGLUT1 puncta, 920 VGAT puncta; small, $n=5$ images, 5462 VGLUT1 puncta, 1455 VGAT puncta) (Fig. 6B). However, when comparing relative size and intensity of excitatory and inhibitory synapses as measured by each punctum's integrated optical density (IOD), we did observe an increase in punctal IOD for inhibitory synapses (1232 \pm 143 large vs $2787 \pm$ 463 small; $p=0.02$ unpaired $t$ test; measured over the same synapses as above) (Fig. 6C). This suggests that inhibitory synapses may also be an important locus of functional scaling as the number of available partners changes.

The relative targeting of excitatory synapses to different postsynaptic cell types was next distinguished by labeling $\alpha$-CaMKII (excitatory dendrites) and GABA (inhibitory dendrites), whereas the excitatory terminals themselves were identified and counted using VGLUT1 (Fig. 6D). Here, the most notable difference between large and small networks was not in the intensity of the synaptic terminals on excitatory dendrites (2157 \pm 146 intensity units/synapse, large vs $2368 \pm 613$, small; $p=0.75$ unpaired $t$ test; large, $n=7$ images, 1455 synapses; small, $n=6$ images, 932 synapses) (Fig. $6 E$, left) or inhibitory dendrites ( $2407 \pm 347$ intensity units/synapse, large vs $2042 \pm 445$, small; $p=0.51$ unpaired $t$ test; large, $n=6$ images, 1400 synapses; small, $n=5$ images, 164 synapses) (Fig. $6 E$, right), nor in the size of VGLUT1-labeled terminals (data not shown), but rather in their density. Specifically, whereas the density of excitatory-toexcitatory projections was similar between large and small networks $(0.0069 \pm 0.0016$ synapses/pixel, large vs $0.0056 \pm 0.0006$ synapses/pixel, small; $p=0.49$ unpaired $t$ test; large, $n=7$ images, 1455 synapses; small, $n=6$ images, 932 synapses) (Fig. $6 F$, left) the density of excitatory-to-inhibitory projections was massively increased in the larger networks $(0.0104 \pm 0.0020$ synapses/pixel, large vs $0.0017 \pm 0.0005$ synapses/pixel, small; $p=$ 0.01 unpaired $t$ test; large, $n=6$ images, 1400 synapses; small, $n=$ 5 images, 164 synapses) (Fig. $6 F$, right), suggesting an amplification of inhibition in these networks that could help to gate network activity by increasing the functional drive to inhibitory subcircuits (Hartman et al., 2006). This structural rearrangement may work together with the scaling of quantal size and functional connectivity (Fig. 5) to redistribute synaptic efficacy throughout the network in support of an increased number of synaptic partners and connections.

\section{Discussion}

We present a system for varying the size of cultured cellular networks on a fine scale, and use it to identify a host of neuronal connectivity parameters that are reconfigured through intrinsic mechanisms after changes in the number of neurons situated in a network.

Although methods for micropatterning cellular substrates are now well worked out (Chen et al., 2000; Whitesides et al., 2001) and have been applied to neurons (Scholl et al., 2000; Dertinger et al., 2002; Sanjana and Fuller, 2004), we believe that the present work is the first to use these techniques to specify network morphology to study properties of synaptic function. Being able to coordinate the size and shape of deposited networks with read- 
Excitatory / Inhibitory Balance:

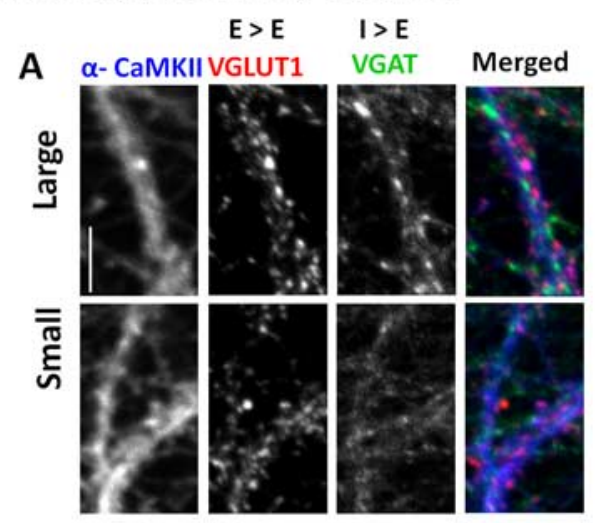

Excitatory Axon Targeting:

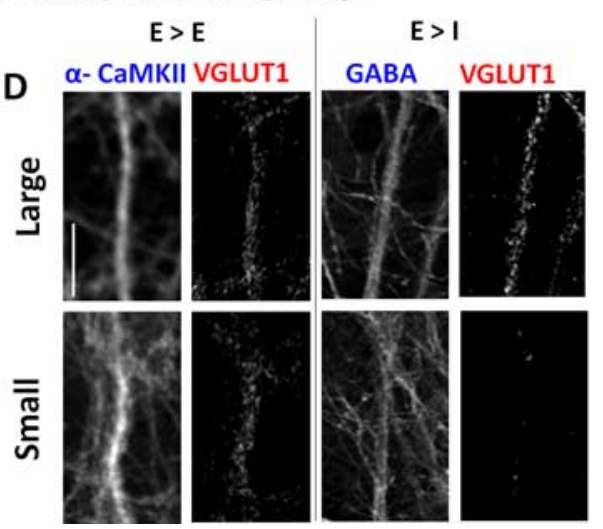

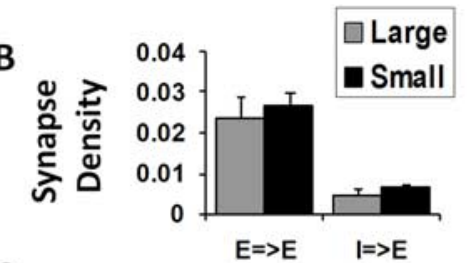

C
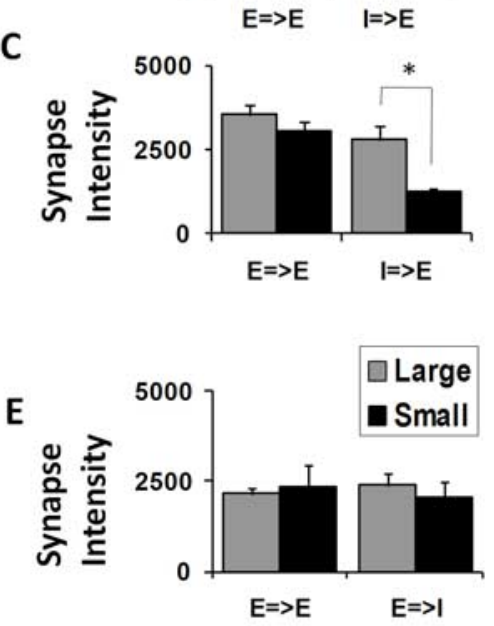

$\mathbf{F}$

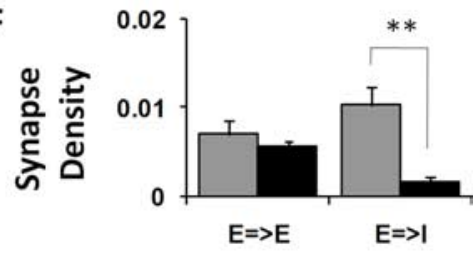

Figure 6. Scaling network size changes functional network architecture and excitatory-inhibitory balance. A, Triple immunostaining for the excitatory dendrite marker $\alpha$-CaMKII, the excitatory synapse marker VGLUT1, and the inhibitory synapse marker VGAT. $\boldsymbol{B}$, Density of excitatory and inhibitory synapses per unit length of excitatory dendrite. There was no significant difference in the relative density of excitatory and inhibitory synapses between large and small networks. $C$, The punctal intensity of excitatory and inhibitory synaptic boutons, as measured by $10 D$, showed no change in the intensity of excitatory synapses from large to small networks. However, the intensity of inhibitory synapses in small networks was significantly decreased compared with those in large networks. D, Triple immunostaining for the excitatory dendrite marker $\alpha$-CaMKII, the inhibitory dendrite marker GABA, and the excitatory synapse marker VGLUT1. $\boldsymbol{E}$, Staining intensity of excitatory synaptic boutons on either excitatory or inhibitory dendrites. No significant difference was detected in excitatory intensity onto either type of postsynaptic cell between large or small networks. $\boldsymbol{F}$, Density of excitatory synapses made onto either an excitatory or inhibitory dendrite in large or small networks. A significant reduction of excitatory synapses onto inhibitory dendrites is observed in small networks. Scale bars: $A, 10 \mu \mathrm{m} ; \mathbf{D}, 20$ $\mu \mathrm{m} .{ }^{*} p<0.05 ;{ }^{* *} p<0.01$. Error bars indicate SEM.

outs of synaptic connectivity and other physiological signatures demonstrates a first step toward engineering customized neuronal networks to explore how network architecture impacts network function.

In our system we observed that when the number of available synaptic partners is increased (Fig. 2), the total number of connections they form is also increased (Fig. 3), whereas total input and activity levels are maintained (Fig. 4) via a redistribution of strength across the connections (Fig. 5) and through a reorganization of connectivity between excitatory and inhibitory neurons (Fig. 6).

Increasing the number of partners available to a neuron increases the number of synapses to each partner

Our result that the number of synapses per partner increases with the number of available partners (Fig. 3) was not necessarily predictable. It could be that neurons are not sensitive to the addition of cellular neighbors, and would conserve their distribution of synapses among a set number of partners even when more are

added, leading to a conserved number of synapses. Or, all other things being equal, neurons could be tuned to spread a conserved number of synapses evenly as potential partners are introduced, such that more and more neurons become connected using fewer and fewer synapses each, again for a conserved number of synapses. Hypothetically, adding more neurons to the recurrent network could even increase the opportunities for spontaneous excitability, thus generating more activity and leading to a homeostatic decrease in the number of synapses. Instead, we find that changing the number of available input partners directly scales synapse number to lead to a more richly connected network, with neurons selecting to scale down synaptic strength in compensation, a result that to our knowledge has no precedent in the literature. Thus, when presented with more potential partners, neurons seemed to trade off fewer, stronger connections for more, weaker ones.

\section{An inverse relationship between number of synaptic partners and unitary synaptic strength}

The tradeoff between a neuron's quantity and "quality" (strength) of synapses as a consequence of scaling is in line with mechanisms intended to redistribute some measure of synaptic efficacy to promote functional stability and/or maximize information processing (Laughlin, 1989; LeMasson et al., 1993; Turrigiano and Nelson, 2000; Davis and Bezprozvanny, 2001; Burrone and Murthy, 2003), and possibly relates to the maintenance of a quantity of aggregate efficacy that is redistributed between a flexible number of partners. Biophysically, this quantity could take the form of postsynaptic AMPA receptors, which are known to undergo fluid trafficking between synapses because of activity-dependent cues (Poncer et al., 2002; Song and Huganir, 2002) and which have been implicated as the ultimate downstream benefactor of "synaptic tagging" protein-synthesis-dependent factors (Frey and Morris, 1997) over which synapses may compete for relative strength (Fonseca et al., 2004).

Our observed changes in quantal size (Fig. $5 A-C$ ) are compatible with a postsynaptic AMPA receptor model, as the size and intensity of a presynaptic molecular correlate of quantal size (VGLUT1) does not change (Fig. 6E). At the same time, an increase in quantal size $(\sim 66 \%)$ (Fig. $5 C$ ) is probably not sufficient to fully explain the $\sim 3.5$ fold increase in overall connection strength that occurs when number of partners is reduced (Fig. 5G); rather, there is probably an additional increase in the probability of release per synapse, or the number of synapses comprising a connection [as in the study by Chen and Regehr (2000)], although the total number of synapses in the network is reduced with the total number of cells that need to be connected (Fig. 3C). Interestingly, multiple aspects of this remodel- 
ing are compatible with a molecular framework proposed by others (Pratt et al., 2003).

\section{Redistribution of excitatory axons}

Labeling excitatory (E) and inhibitory (I) neurons and synapses simultaneously (Fig. 6), we checked interactions between cell types that might undergo structural reconfiguration as the size of the network was scaled. We found that between $\mathrm{E} \rightarrow \mathrm{E}$ synapses, $\mathrm{I} \rightarrow$ E synapses, and $\mathrm{E} \rightarrow \mathrm{I}$ synapses, it was the $\mathrm{E} \rightarrow \mathrm{I}$ synapses (excitatory neurons synapsing onto inhibitory ones) that appeared most impacted structurally. Excitatory axons that gave rise to either $\mathrm{E} \rightarrow \mathrm{E}$ synapses or $\mathrm{E} \rightarrow$ I ones increasingly chose $\mathrm{E} \rightarrow \mathrm{I}$ ones as the size of the network scaled. The purpose of this could be to allow the excitatory neurons to use the targeting of their axons to elevate their own inhibition to accommodate a surplus of excitatory input. Turning to inhibitory loops would enable this accommodation without disrupting a local excitatory/inhibitory structural balance in synapse number on the excitatory dendrite (Liu, 2004). This is also consistent with the view that stable representations reside not only in single excitatory cells, but within balanced interactions between excitatory and inhibitory neurons (Somers et al., 1998; Palmer and Miller, 2007).

\section{Implications for synaptic plasticity during aging and disease states}

It is believed that during aging and disease states such as Alzheimer's Disease, the number of neurons in specific regions such as the hippocampus undergoes a profound reduction (Coleman and Flood, 1987; Simic et al., 1997). Interestingly, this is also accompanied by a reduction in the number of functional synapses (Barnes et al., 1992; Geinisman et al., 1992; Rosenzweig and Barnes, 2003), and yet at least in aging the average activity levels are mostly unchanged (Rao et al., 1993; Tanila et al., 1997; Smith et al., 2000), thus mimicking part of the phenomena that we observe in our neuronal clusters (Figs. 2-4). Potentially, it is the neurons' intrinsic capacity to redistribute synaptic efficacy to their remaining contacts which enables them to preserve their activity levels as the size of the network shrinks. In turn, it has also been noted that stronger synapses are often associated with reduced plasticity (Bi and Poo, 1998; Slutsky et al., 2004). If neuronal and synaptic loss were compensated for by a reconfiguration of the remaining inputs, and surviving neurons salvage their firing rates by strengthening their remaining synapses, the plasticity of those synapses may be compromised. Thus, synaptic scaling and reconfiguration as a result of neuron loss could contribute to known age-related deficits in synaptic plasticity (Rosenzweig and Barnes, 2003) or impact specific regions during diseases where neuron number is suspected to change, such as in autism (Schumann and Amaral, 2006). As a consequence, although neuron number is projected to be somewhat redundant in the brain, physiological parameters that are set according to that number could be impacted as neurons are pathologically lost.

Implications for development and representational plasticity During and after development, the size of network representations continues to scale throughout an organism's life, with experience and practice. However, as network representations grow to be larger, individual neurons will actually account for a smaller piece of the total representation (Hubel and Wiesel, 1974; Sur et al., 1980). Interestingly, here a synaptic redistribution according to network size would lead neurons to tune their inputs down, in keeping with each input's newly reduced functional relevance. The capacity to redistribute synaptic efficacy in this manner would enable neurons to sample and consolidate input from a varying number of neurons without changing absolute activity levels (Markram and Tsodyks, 1996). Exploring the molecular underpinnings of this circuit reconfiguration phenomenon, and its potential implementation during development, experience, and aging, will likely reveal deeper insights into its purpose and organizing role.

\section{References}

Barnes CA, McNaughton BL (1980) Physiological compensation for loss of afferent synapses in rat hippocampal granule cells during senescence. J Physiol (Lond) 309:473-485.

Barnes CA, Rao G, Foster TC, McNaughton BL (1992) Region-specific age effects on AMPA sensitivity: electrophysiological evidence for loss of synaptic contacts in hippocampal field CA1. Hippocampus 2:457-468.

Betz WJ, Bewick GS (1992) Optical analysis of synaptic vesicle recycling at the frog neuromuscular junction. Science 255:200-203.

Bi GQ, Poo MM (1998) Synaptic modifications in cultured hippocampal neurons: dependence on spike timing, synaptic strength, and postsynaptic cell type. J Neurosci 18:10464-10472.

Burrone J, Murthy VN (2003) Synaptic gain control and homeostasis. Curr Opin Neurobiol 13:560-567.

Chen C, Regehr WG (2000) Developmental remodeling of the retinogeniculate synapse. Neuron 28:955-966.

Chen CS, Ostuni E, Whitesides GM, Ingber DE (2000) Using self-assembled monolayers to pattern ECM proteins and cells on substrates. Methods Mol Biol 139:209-219.

Coleman PD, Flood DG (1987) Neuron numbers and dendritic extent in normal aging and Alzheimer's disease. Neurobiol Aging 8:521-545.

Colman H, Nabekura J, Lichtman JW (1997) Alterations in synaptic strength preceding axon withdrawal. Science 275:356-361.

Davis GW, Goodman CS (1998) Synapse-specific control of synaptic efficacy at the terminals of a single neuron. Nature 392:82-86.

Davis GW, Bezprozvanny I (2001) Maintaining the stability of neural function: a homeostatic hypothesis. Annu Rev Physiol 63:847-869.

Dertinger SK, Jiang X, Li Z, Murthy VN, Whitesides GM (2002) Gradients of substrate-bound laminin orient axonal specification of neurons. Proc Natl Acad Sci USA 99:12542-12547.

Fonseca R, Nagerl UV, Morris RG, Bonhoeffer T (2004) Competing for memory: hippocampal LTP under regimes of reduced protein synthesis. Neuron 44:1011-1020.

Foster TC, Barnes CA, Rao G, McNaughton BL (1991) Increase in perforant path quantal size in aged F-344 rats. Neurobiol Aging 12:441-448.

Frey U, Morris RG (1997) Synaptic tagging and long-term potentiation. Nature 385:533-536.

Geinisman Y, de Toledo-Morrell L, Morrell F, Persina IS, Rossi M (1992) Age-related loss of axospinous synapses formed by two afferent systems in the rat dentate gyrus as revealed by the unbiased stereological dissector technique. Hippocampus 2:437-444.

Hartman KN, Pal SK, Burrone J, Murthy VN (2006) Activity-dependent regulation of inhibitory synaptic transmission in hippocampal neurons. Nat Neurosci 9:642-649.

Hubel DH, Wiesel TN (1974) Uniformity of monkey striate cortex: a parallel relationship between field size, scatter, and magnification factor. J Comp Neurol 158:295-305.

Jones EG, Huntley GW, Benson DL (1994) Alpha calcium/calmodulindependent protein kinase II selectively expressed in a subpopulation of excitatory neurons in monkey sensory-motor cortex: comparison with GAD-67 expression. J Neurosci 14:611-629.

Laughlin SB (1989) The role of sensory adaptation in the retina. J Exp Biol 146:39-62.

LeMasson G, Marder E, Abbott LF (1993) Activity-dependent regulation of conductances in model neurons. Science 259:1915-1917.

Lichtman JW, Colman H (2000) Synapse elimination and indelible memory. Neuron 25:269-278

Liu G (2004) Local structural balance and functional interaction of excitatory and inhibitory synapses in hippocampal dendrites. Nat Neurosci 7:373-379.

Liu G, Tsien RW (1995a) Properties of synaptic transmission at single hippocampal synaptic boutons. Nature 375:404-408.

Liu G, Tsien RW (1995b) Synaptic transmission at single visualized hippocampal boutons. Neuropharmacology 34:1407-1421. 
Liu XB, Jones EG (1996) Localization of alpha type II calcium calmodulindependent protein kinase at glutamatergic but not gamma-aminobutyric acid (GABAergic) synapses in thalamus and cerebral cortex. Proc Natl Acad Sci USA 93:7332-7336.

Markram H, Tsodyks M (1996) Redistribution of synaptic efficacy: a mechanism to generate infinite synaptic input diversity from a homogeneous population of neurons without changing absolute synaptic efficacies. J Physiol Paris 90:229-232.

Palmer SE, Miller KD (2007) Effects of inhibitory gain and conductance fluctuations in a simple model for contrast-invariant orientation tuning in cat v1. J Neurophysiol 98:63-78.

Poncer JC, Esteban JA, Malinow R (2002) Multiple mechanisms for the potentiation of AMPA receptor-mediated transmission by $\alpha-\mathrm{Ca}^{2+}$ / calmodulin-dependent protein kinase II. J Neurosci 22:4406-4411.

Pratt KG, Watt AJ, Griffith LC, Nelson SB, Turrigiano GG (2003) Activitydependent remodeling of presynaptic inputs by postsynaptic expression of activated CaMKII. Neuron 39:269-281.

Rao G, Barnes CA, McNaughton BL (1993) Effects of age on L-glutamateinduced depolarization in three hippocampal subfields. Neurobiol Aging 14:27-33.

Rosenzweig ES, Barnes CA (2003) Impact of aging on hippocampal function: plasticity, network dynamics, and cognition. Prog Neurobiol 69:143-179.

Sanjana NE, Fuller SB (2004) A fast flexible ink-jet printing method for patterning dissociated neurons in culture. J Neurosci Methods 136:151-163.

Scholl M, Sprossler C, Denyer M, Krause M, Nakajima K, Maelicke A, Knoll W, Offenhausser A (2000) Ordered networks of rat hippocampal neurons attached to silicon oxide surfaces. J Neurosci Methods 104:65-75.

Schumann CM, Amaral DG (2006) Stereological analysis of amygdala neuron number in autism. J Neurosci 26:7674-7679.

Sik A, Hajos N, Gulacsi A, Mody I, Freund TF (1998) The absence of a major $\mathrm{Ca}^{2+}$ signaling pathway in GABAergic neurons of the hippocampus. Proc Natl Acad Sci USA 95:3245-3250.
Simic G, Kostovic I, Winblad B, Bogdanovic N (1997) Volume and number of neurons of the human hippocampal formation in normal aging and Alzheimer's disease. J Comp Neurol 379:482-494.

Singhvi R, Kumar A, Lopez GP, Stephanopoulos GN, Wang DI, Whitesides GM, Ingber DE (1994) Engineering cell shape and function. Science 264:696-698.

Sloviter RS, Dichter MA, Rachinsky TL, Dean E, Goodman JH, Sollas AL, Martin DL (1996) Basal expression and induction of glutamate decarboxylase and GABA in excitatory granule cells of the rat and monkey hippocampal dentate gyrus. J Comp Neurol 373:593-618.

Slutsky I, Sadeghpour S, Li B, Liu G (2004) Enhancement of synaptic plasticity through chronically reduced $\mathrm{Ca}^{2+}$ flux during uncorrelated activity. Neuron 44:835-849.

Smith AC, Gerrard JL, Barnes CA, McNaughton BL (2000) Effect of age on burst firing characteristics of rat hippocampal pyramidal cells. NeuroReport 11:3865-3871.

Somers DC, Todorov EV, Siapas AG, Toth LJ, Kim DS, Sur M (1998) A local circuit approach to understanding integration of long-range inputs in primary visual cortex. Cereb Cortex 8:204-217.

Song I, Huganir RL (2002) Regulation of AMPA receptors during synaptic plasticity. Trends Neurosci 25:578-588.

Sur M, Merzenich MM, Kaas JH (1980) Magnification, receptive-field area, and "hypercolumn" size in areas $3 \mathrm{~b}$ and 1 of somatosensory cortex in owl monkeys. J Neurophysiol 44:295-311.

Sur M, Weller RE, Sherman SM (1984) Development of X- and Y-cell retinogeniculate terminations in kittens. Nature 310:246-249.

Tanila H, Shapiro M, Gallagher M, Eichenbaum H (1997) Brain aging: changes in the nature of information coding by the hippocampus. J Neurosci 17:5155-5166.

Turrigiano GG, Nelson SB (2000) Hebb and homeostasis in neuronal plasticity. Curr Opin Neurobiol 10:358-364.

Whitesides GM, Ostuni E, Takayama S, Jiang X, Ingber DE (2001) Soft lithography in biology and biochemistry. Annu Rev Biomed Eng 3:335373 . 